\title{
Multi-area Coordination of Security-Constrained Dynamic Optimal Power Flow in AC-DC Grids with Energy Storage
}

\author{
Nico Huebner, Nils Schween, Michael Suriyah, Vincent Heuveline, \\ and Thomas Leibfried
}

\begin{abstract}
In times of growing integrated electricity markets and needed coordination of large inter-regional physical power flows, multi-area Optimal Power Flow (OPF), also referred to as distributed OPF, has gained importance in research. However, the conventional OPF is only of limited use since a TSO is strongly interested in N-1 security. Furthermore, time-dependent constraints such as generator ramping or energy storage limits play a growing role. Consequently, a Security-Constrained Dynamic OPF (SC-D-OPF) includes both N-1 security and quasi-stationary dynamics. We present a decoupling approach to compute an SCD-OPF by coordination among inter-connected areas. Privacy is maintained by implementing an Alternating Direction of Multipliers Method (ADMM), where only results of boundary variables are exchanged with a neighbor. We show the functionality of the approach in a small test case, where the distributed result is close to that of a centralized optimization.
\end{abstract}

Keywords Multi-area optimal power flow - Distributed optimal power flow $\cdot$ N-1 security · Energy storage - Dynamic optimal power flow $\cdot$ Multi-period optimal power flow $\cdot$ Embedded HVDC $\cdot$ AC-DC grids

\footnotetext{
N. Huebner $(\bowtie) \cdot$ M. Suriyah · T. Leibfried Institute of Electric Energy Systems and High-Voltage Technology (IEH), Karlsruhe Institute of Technology (KIT), Karlsruhe, Germany

e-mail: nico.meyer-huebner@kit.edu

N. Schween · V. Heuveline

Engineering Mathematics and Computing Lab (EMCL), Interdisciplinary Center for Scientific Computing (IWR), Heidelberg, Germany
}

V. Bertsch et al. (eds.), Advances in Energy System Optimization,

Trends in Mathematics, https://doi.org/10.1007/978-3-030-32157-4_3 


\section{Introduction}

The increasing penetration of Renewable Energy Sources (RES) leads to a power system operation closer to its operational limits. Enhanced methods and tools will be crucial for a secure, but also efficient and cheap planning and operation of the power grid. A Transmission System Operator (TSO) will have larger assets of controllable devices at hand, such as Voltage Source Converter (VSC-) based HVDC-systems and energy storage systems. Combined with the need to assure N-1 security, this requires a Security-Constrained Dynamic Optimal Power Flow (SC-D-OPF), which incorporates both $\mathrm{N}-1$ security and multiple time steps into the optimization. First research in that area was done in [1], where not only power but also necessary energy reserves can be determined to ensure N-1 security. In [2], successive linear algorithms and approximated power flows are used to solve large-scale SC-D-OPF problems in a European context. The authors of [3] propose an SC-D-OPF model including uncertainty of wind power or equipment availability, which is solved in a two-stage stochastic program. In [4], SC-D-OPF is used as inner iteration in a hierarchical approach to optimize a central deployment signal which is sent to the units able to provide a reserve. Furthermore, [5-7] include energy storage systems in an SC-D-OPF calculation. A further extension is shown in [8], where SC-D-OPF is solved in a hybrid AC-DC grid with energy storage.

Due to the increasing complexity of the power system and an operation closer to network limitations, central coordination in large scale networks comes with major computational burdens. Furthermore, privacy can be a concern for each transmission system operator (TSO) controlling a certain region. Subsequently, the interest in distributed optimization, also referred to as multi-area optimization has substantially grown in recent years. An early overview of distributed OPF algorithms can be found in [9] and the most recent developments are examined in detail in [10]. The Alternating Direction of Multipliers Method (ADMM) [11], has become a popular branch to tackle the non-convex AC OPF problem [12-14]. Each area uses variable duplicates from neighboring areas and is solved to optimality. Penalty terms, which are calculated from the coupling variable deviation and Lagrangian multipliers, are added to the objective function to push two neighbors towards a common boundary solution. ADMM was applied to a hybrid AC-DC grid in [15]. Multiarea optimization is applied to D-OPF with storage in [16] using OCD, but to the best of our knowledge, no attempts have been made toward distributing SC-D-OPF.

\section{Security-Constrained Dynamic Optimal Power Flow (SC-D-OPF)}

The optimization horizon is defined by a set of time steps $\mathcal{T}=\{1, \ldots, T\}$ with $T$ the total number of considered time steps. Furthermore, we define a scenario set $\mathcal{C}=\{0, \ldots, C\}$. Here, scenario $c=0$ defines the base case, i.e. the original fault- 
free network. A total of $C$ possible contingencies is added by including modified versions of the base case to the scenario set. A modification could be the outage of an AC line, a DC line or a converter. With $x^{t, c}$ the optimization variables of time step $t$ and scenario $c$, the full set of optimization variables is collected in

$$
x=\left(\left(x^{1,0}\right)^{\top}, \ldots,\left(x^{1, C}\right)^{\top}, \ldots,\left(x^{T, 0}\right)^{\top}, \ldots,\left(x^{T, C}\right)^{\top}\right)^{\top} .
$$

The full problem has the form (2):

$$
\begin{array}{cl}
\underset{x}{\operatorname{minimize}} & \sum_{t \in \mathcal{T}} \sum_{c \in \mathcal{C}} p^{t, c} \cdot f\left(x^{t, c}\right) \\
\text { subject to } & h_{\text {base }}\left(x^{t, c}\right) \leq 0 \quad \forall t \in \mathcal{T}, c \in \mathcal{C} \\
& h_{\text {dyn }}\left(x^{t, 0}, x^{t-1,0}\right) \leq 0 \quad \forall t \in \mathcal{T} \backslash 1 \\
& h_{\mathrm{N}-1}\left(x^{t, 0}, x^{t, c}\right) \leq 0 \quad \forall t \in \mathcal{T}, c \in \mathcal{C} \backslash 0 .
\end{array}
$$

It minimizes the sum of weighted costs $f$ over all scenarios. Factor $p^{t, c}$ defines a probability of each contingency to enable a risk-based OPF. Constraints $h_{\text {base }}$ describe the basic constraints of a conventional OPF, which must be fulfilled during each scenario $(t, c)$. That includes $\mathrm{AC}$ and $\mathrm{DC}$ node power balance; thermal AC and DC branch limits; AC and DC voltage limits; quadratic loss function of ACDC converters; and operational limits of generators, converters or sheddable loads. Time-dependent constraints, such as storage energy limits or generator ramping limits, are collected in $h_{\text {dyn }}$. Those constraints only apply to the base case $(c=0)$, since set points after an outage are coupled solely to the respective base case of the identical time step. This $\mathrm{N}-1$ coupling is modeled by the constraints $h_{\mathrm{N}-1}$ and guarantees N-1 security. Here, the coupling between each contingency and the base case is explicitly modeled. That is, set point deviation limits from before to after the occurrence of an outage can be defined. Note that for the sake of readability, we omit equality constraints which can be interpreted as reformulated inequalities as well. We refer to [8] for more details on the modeling.

\section{Distributed Formulation}

For the sake of readability, we write (2) in the form (3):

$$
\begin{array}{ll}
\underset{x}{\operatorname{minimize}} & f(x) \\
\text { subject to } & h(x) \leq 0 .
\end{array}
$$


Let $R$ non-overlapping regions be defined in $\mathcal{R}=\{1, \ldots, R\}$, that is, each node belongs to exactly one region. An equivalent problem as (3), but in separable form, can be written as (4):

$$
\begin{array}{ll}
\underset{x_{k}, \forall k \in \mathcal{R}}{\operatorname{minimize}} & \sum_{k \in \mathcal{R}} f_{k}\left(x_{k}\right) \\
\text { subject to } & h_{k}\left(x_{k}\right) \leq 0, \quad \forall k \in \mathcal{R} \\
& \sum_{k \in \mathcal{R}} A_{k} x_{k}=0 .
\end{array}
$$

Here, $x_{k} \in \mathbb{R}^{l_{k}}, h_{k}: \mathbb{R}^{l_{k}} \rightarrow \mathbb{R}^{m_{k}}$ and $f_{k}: \mathbb{R}^{l_{k}} \rightarrow \mathbb{R}$ are optimization variables, non-linear constraints and objective function, respectively, in a region $k$. Matrix $A_{k} \in \mathbb{R}^{n \times l_{k}}$ maps $x_{k}$ onto the full set of $n$ coupling constraints and enforces consensus between regions. Thus, (4b) forms independent local SC-DOPF constraints and (4c) form the coupling constraints which recuperate a feasible overall power flow.

\section{Network Decomposition in AC-DC Grids}

To allow for a separable formulation, the network is first partitioned and then decomposed.

Network partitioning can be crucial for distributed algorithms to achieve good performance. We believe that network partitions are inherently given by structural responsibilities. For example, a region or control area could represent one TSO, multiple TSOs or a whole country. Thus, the number and dimension of AC regions are historically known. However, responsibilities in overlaying DC networks are yet to be defined and various options are thinkable [15]. We choose a Shared-DC approach. Here, we define $R=R^{\mathrm{AC}}$ hybrid AC-DC regions, where each AC region may also contain DC nodes. Thus, each existing TSO gains control over converters in its own control area. Let $\mathcal{N}_{k}$ identify all nodes in region $k$. Herewith, $\mathcal{N}_{k}^{\mathrm{AC}}$ collects all $\mathrm{AC}$ nodes and $\mathcal{N}_{k}^{\mathrm{DC}}$ all DC nodes in region $k$.

\subsection{Decoupling of AC or DC Tie Line}

In principle, the decoupling of AC and DC tie line is identical, except for extended consensus constraints due to complex voltage and power in the AC case (Fig. 1).

The original line is cut into two halves; auxiliary nodes $(m, n)$ and auxiliary generators $(a, b)$ are added at both open ends. Thus, node sets are augmented to $\mathcal{N}_{A} \leftarrow\left\{\mathcal{N}_{A}, m\right\}, \mathcal{N}_{B} \leftarrow\left\{\mathcal{N}_{B}, n\right\}$. To guarantee a feasible power flow, the voltage 


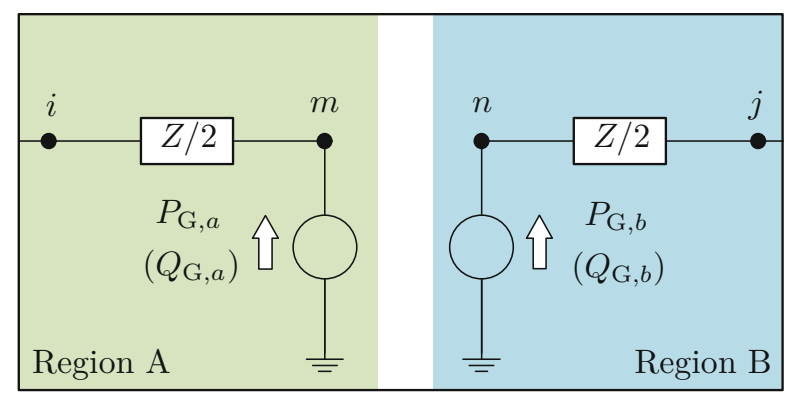

Fig. 1 Decoupling model of a tie line (AC or DC) between nodes $i$ and $j$. Two auxiliary nodes $(m$ and $n$ ) and two auxiliary generators $\left(a\right.$ and $b$ ) are added at the middle. Reactive power source $Q_{\mathrm{G}}$ is only added for an AC tie line

must be equal at nodes $m$ and $n$. Furthermore, the generators must produce the same amount of power of the opposite sign. In the case of an AC tie line $\left(i \in \mathcal{N}_{A}^{\mathrm{AC}}, j \in\right.$ $\mathcal{N}_{B}^{\mathrm{AC}}$ ), this leads to boundary conditions including complex voltage and both active and reactive power. For time step and scenario $(t, c)$, we have:

$$
\begin{aligned}
\left|V_{\mathrm{AC}, m}^{t, c}\right| & =\left|V_{\mathrm{AC}, n}^{t, c}\right| \\
\angle V_{\mathrm{AC}, m}^{t, c} & =\angle V_{\mathrm{AC}, n}^{t, c} \\
P_{\mathrm{G}, a}^{t, c} & =-P_{\mathrm{G}, b}^{t, c} \\
Q_{\mathrm{G}, a}^{t, c} & =-Q_{\mathrm{G}, b}^{t, c} .
\end{aligned}
$$

In the case of a DC tie line $\left(i \in \mathcal{N}_{A}^{\mathrm{DC}}, j \in \mathcal{N}_{B}^{\mathrm{DC}}\right.$ ), only real voltage and active power must meet the constraints. For time step and scenario $(t, c)$, we have:

$$
\begin{aligned}
V_{\mathrm{DC}, m}^{t, c} & =V_{\mathrm{DC}, n}^{t, c} \\
P_{\mathrm{G}, a}^{t, c} & =-P_{\mathrm{G}, b}^{t, c} .
\end{aligned}
$$

Equations (5) and (6) are the only boundary constraints.

\subsection{Building Consensus Constraint Matrix A}

Since $x_{k}^{t, c}$ uses the augmented node sets of region $k$, the auxiliary generator variables are included. Thus, consensus constraints (5)-(6) between region $A$ and $B$ can be written to

$$
\tilde{A}_{A}^{t, c} x_{\mathrm{A}}^{t, c}+\tilde{A}_{B}^{t, c} x_{\mathrm{B}}^{t, c}=0 .
$$




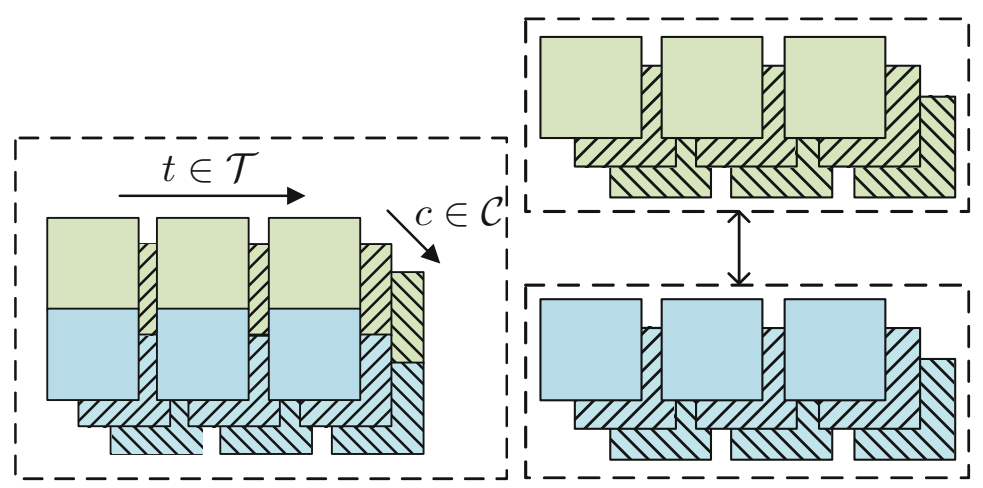

(a)

(b)

Fig. 2 Schematic problem decomposition for two areas (blue and green) under consideration of three time steps and two contingencies. A dashed box represents an optimization problem. (a) Centralized SC-D-OPF. (b) Distributed SC-D-OPF

This must be fulfilled for every considered time or contingency scenario (Fig. 2). Then we have

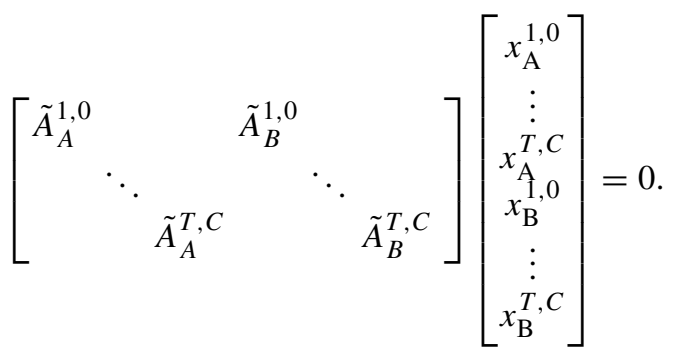

In a more compact form, (8) can be written as

$$
\sum_{k \in\{A, B\}} A_{k} x_{k}=0
$$

\section{Implemented ADMM Algorithm}

The general idea of ADMM, see also [11], is the following. Augmented regional OPFs are solved and the deviation of boundary variables from a fixed auxiliary variable $z$, which is information stemming from neighboring regions, is penalized. The regions then exchange information and $z$ is updated. The update is re-distributed to the local agents (areas) for a new OPF calculation until consensus between 
regions is achieved. It is constructed an augmented Lagrangian of the form

$$
\begin{aligned}
\mathcal{L}(x, z, \lambda) & =\sum_{k \in \mathcal{R}}\left\{f_{k}\left(x_{k}\right)+\lambda_{k}^{\top} A_{k}\left(x_{k}-z_{k}\right)\right. \\
& \left.+\frac{\rho}{2}\left\|A_{k}\left(x_{k}-z_{k}\right)\right\|_{W}^{2}\right\},
\end{aligned}
$$

with penalty parameter $\rho \in \mathbb{R}$. Dual variables of the consensus constraints are denoted with $\lambda_{k} \in \mathbb{R}^{n \times 1}$, and $W \in \mathbb{R}^{n \times n}$ is a positive definite, diagonal weighting matrix, ${ }^{1}$ where each entry is related to one coupling constraint. We refer to $W(S)$ if the entry is related to a power variable, and to $W(V)$ if the entry is related to a voltage variable. In ADMM literature, $W$ is the identity matrix. The main steps during one iteration of the solving process are

$$
\begin{aligned}
& \text { 1. } x=\operatorname{argmin}_{x \in \mathcal{X}} \mathcal{L}(x, z, \lambda) \\
& \text { 2. } z=\operatorname{argmin}_{z \in \mathcal{Z}} \mathcal{L}(x, z, \lambda) \\
& \text { 3. } \lambda \leftarrow \lambda+\rho W A(x-z)
\end{aligned}
$$

with $z=\left[\begin{array}{lll}z_{1}^{\top} & \ldots & z_{R}^{\top}\end{array}\right]^{\top}$. The first step (11a) minimizes a non-linear problem, where constraint region

$$
\mathcal{X}=\left\{x \mid h_{k}\left(x_{k}\right) \leq 0, \forall k \in \mathcal{R}\right\}
$$

enforces local constraints (4b). Since $z$ is fixed, (11a) is in fact a series of $R$ independent problems which can be calculated in parallel. The second step minimizes a coupled quadratic problem, where

$$
\mathcal{Z}=\left\{z \mid \sum_{k \in \mathcal{R}} A_{k} z_{k}=0\right\}
$$

enforces consensus of auxiliary variables $z$. In fact, (11b) calculates the average value between two consensus variables of neighboring regions $[11,13]$ and the problem can be reduced to

$$
z=\operatorname{argmin}_{z \in \mathcal{Z}} \sum_{k \in \mathcal{R}} \frac{\rho}{2}\left\|A_{k}\left(x_{k}-z_{k}\right)\right\|_{W}^{2} .
$$

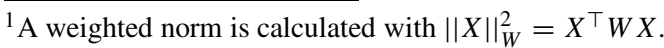




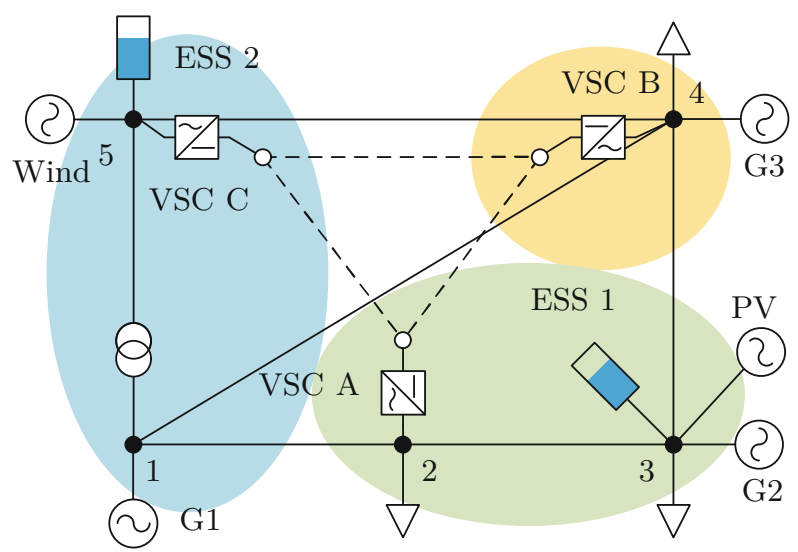

Fig. 3 Test system with embedded HVDC, energy storage and RES. Partitioning into 3 control areas

The weighting factors $\rho$ and $W$ can be neglected in (14), if the same weight is assigned to two coupled variables, which is a reasonable choice. Once a region has gathered necessary neighbor information, this step can be calculated locally as well [12]. In the third step (11c), dual variables are updated based on a weighted distance between $x$ and $z$. Again, with given local $x_{k}$ and $z_{k}$, each region can calculate $\lambda_{k}$ independently (Fig. 3).

In ADMM, an update rule on $\rho$ can be useful to enforce consensus. A $\rho_{k} \in \mathbb{R}$ is assigned to each region, which can be increased depending on the local residual, see (18b). If the residual has not decreased sufficiently compared to the previous iteration (indicator $0<\Theta \in \mathbb{R}<1$ ), the penalty is increased by a constant factor of $\tau \in \mathbb{R}>1$. The penalty parameter must be chosen carefully since it is widely known to be crucial for good convergence behavior [17]. An overview of the implemented ADMM is given in Algorithm 1.

\section{Results}

\subsection{Test Scenario}

We use the illustrative AC-DC test system from [15], see Tables 1 and 2 for line and generator parameters, respectively. The generator at Node 5 is interpreted as a wind park and a PV park is connected to Node 3. RES upper power limit and load are variable over time. Forecast power profiles are taken from the Belgian transmission system operator Elia, which provides detailed RES generation and load data on its website. Adapted profiles for usage in the 5-bus test system are shown in Figs. 6 and 7. We assume quadratic generator cost functions as in [18] (see Table 3) and one 


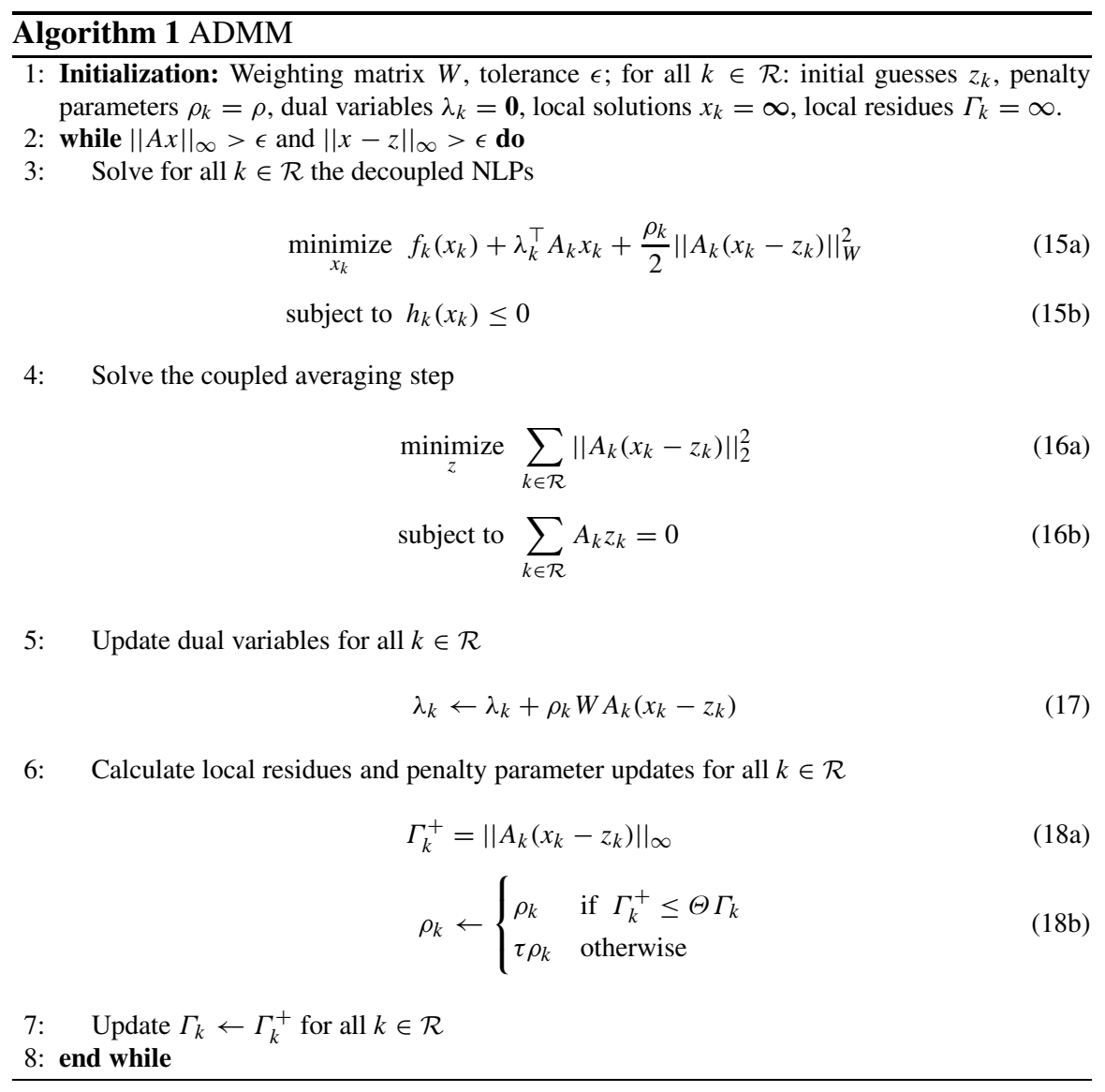

cost coefficient for all reactive power injections. RES power injection is prioritized by assigning a low fuel price. Two energy storage systems with characteristics from Table 4 are connected to Node 3 and 5, respectively. Line flow limits of 400 MVA for Line 1-2 and 240 MVA for Line 4-5, respectively, are used. Furthermore, the capacity of each VSC is set to 200 MVA. Allowed voltage ranges lie between 0.9 and $1.1 \mathrm{pu}$ on the $\mathrm{AC}$ side, and between 0.95 and $1.05 \mathrm{pu}$ on the $\mathrm{DC}$ side.

\subsection{Multi-area SC-D-OPF}

To show applicability of the distributed approach to $\mathrm{N}-1$ secure and dynamic OPF problems, a small example is presented in the following. An SC-D-OPF dispatch optimization is performed for the scenario set $\mathcal{C}=\{0$, Line $1-2\}$ and $\mathcal{T}=\{1, \ldots, 4\}$. Storages and VSCs are allowed curative control, that is, operating 

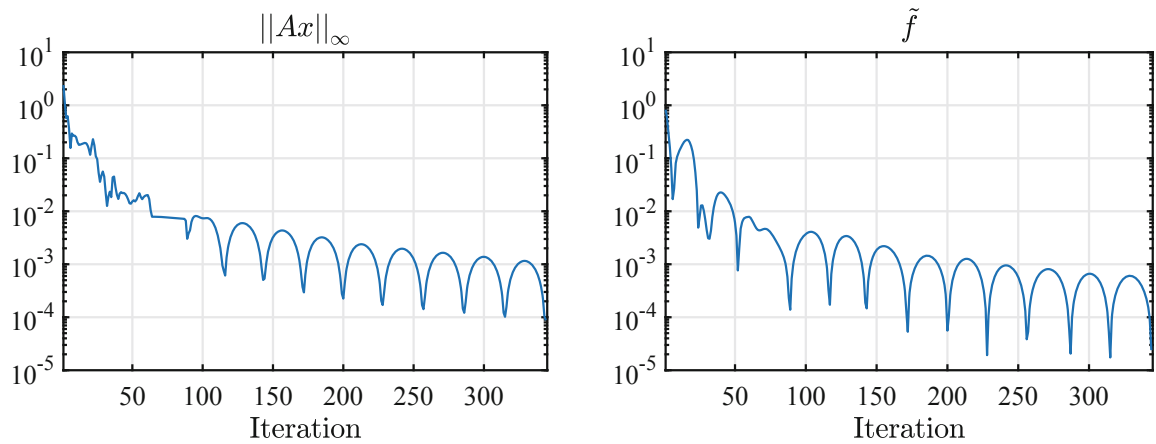

Fig. 4 Convergence behavior of distributed SC-D-OPF in the 5-bus system with 4 time steps and 1 contingency. Left: consensus error, right: cost error. Both relative to central solution

set points are allowed to move freely to a new set point after an outage. Contrarily, conventional generators are operated preventively, that is, the power set point must be valid both before and after the outage. However, to cope with changing system losses after an outage, a deviation of $1 \%$ of installed capacity is allowed.

We use ADMM settings $\rho=500, \tau=1.02, \Theta=0.99, W(S)=1$ and $W(V)=$ 100.

General convergence behavior is shown in Fig. 4. Consensus error and objective suboptimality show similar behavior compared to conventional OPF cases. Consensus error $\left(\|A x\|_{\infty}\right)$ which depicts cross-border feasibility, falls below the threshold of $10^{-4}$ after 344 iterations. The deviation from centrally computed costs $f^{*}$ is denoted with $\tilde{f}=\left|1-f / f^{*}\right|$. The objective value error falls below $0.01 \%$ when convergence is achieved.

The progress of storage variables is shown in Fig. 5 for all time steps after the outage. Dashed lines denote results from the centralized solution. Storage systems provide positive (ESS 2) and negative (ESS 1) power reserve after the outage. The amount of reserve is increased with time for a growing network stress level, but energy levels remain within limits. The distributed solution is represented by solid lines. Those oscillate around and eventually approach the target levels.

\section{Conclusion}

This paper presents an approach to calculate SC-D-OPF in a distributed manner. That is, each control area computes a local N-1 secure and dynamic optimal dispatch which respects boundary constraints from neighboring areas for each time step and topology. After iteratively exchanging information and updating the local solution, a consensus is found which allows for a feasible cross-border power flow for all possible scenarios. Additionally, the result is close to the central optimal solution. Optimal curative storage or VSC set points can be determined for a contingency 

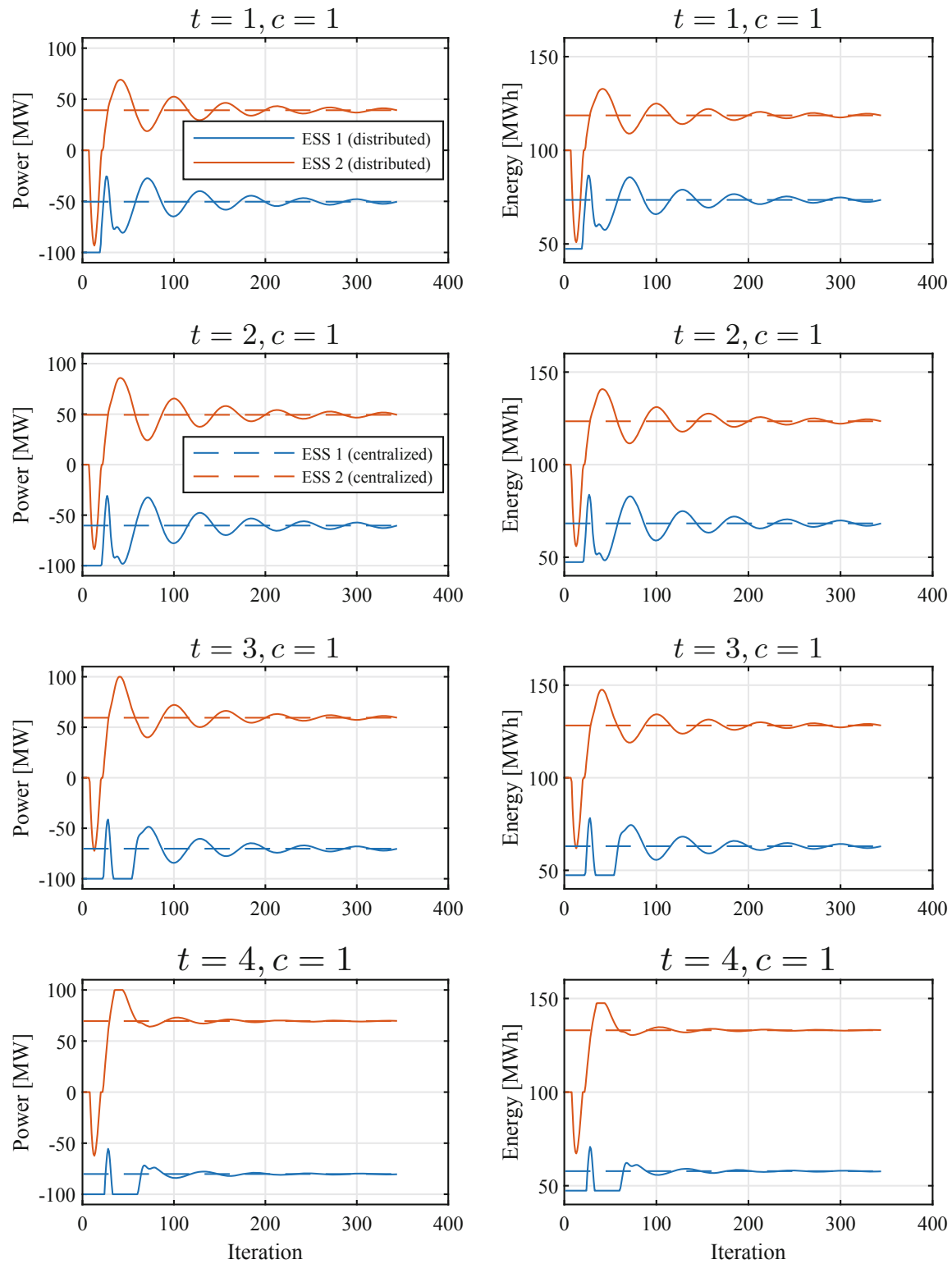

Fig. 5 Convergence of storage power and energy levels after the outage 
scenario which is in a foreign area. In this work, we apply the method to a small test system - the next steps are to increase system size, time horizon and number of contingencies.

\section{Appendix}

Table 1 Line parameters with base power $100 \mathrm{MVA}$

Table 2 Generator parameters. $\left(\underline{P_{\mathrm{G}}}, \overline{P_{\mathrm{G}}}\right)$ in $[\mathrm{MW}]$ and $\left(\overline{Q_{\mathrm{G}}}, \overline{Q_{\mathrm{G}}}\right)$ in [Mvar]

Table 3 Generator cost coefficients

Table 4 Parameters for each energy storage system

\begin{tabular}{l|l|l|l}
\hline Line & $R[\mathrm{pu}]$ & $X[\mathrm{pu}]$ & Total $B[\mathrm{pu}]$ \\
\hline $1-2$ & 0.00281 & 0.0281 & 0.00712 \\
\hline $1-4$ & 0.00304 & 0.0304 & 0.00658 \\
\hline $1-5$ & 0.00064 & 0.0064 & 0.03126 \\
\hline $2-3$ & 0.00108 & 0.0108 & 0.01852 \\
\hline $4-3$ & 0.00297 & 0.0297 & 0.00674 \\
\hline $5-4$ & 0.00297 & 0.0297 & 0.00674 \\
\hline DC & 0.0002 & 0 & 0 \\
\hline
\end{tabular}

\begin{tabular}{l|l|l|l|l|l}
\hline Generator & Node & $P_{\mathrm{G}}$ & $\overline{P_{\mathrm{G}}}$ & $Q_{\mathrm{G}}$ & $\overline{Q_{\mathrm{G}}}$ \\
\hline $\mathrm{G} 1$ & 1 & 0 & 170 & -127.5 & 127.5 \\
\hline $\mathrm{G} 2$ & 3 & 0 & 520 & -390 & 390 \\
\hline $\mathrm{G} 3$ & 4 & 0 & 200 & -150 & 150 \\
\hline
\end{tabular}

\begin{tabular}{l|l|l|l}
\hline & & \multicolumn{3}{|l}{ Cost coefficients } \\
\hline Generator & $a_{\mathrm{G}}\left[\frac{1}{\mathrm{MW}^{2}} \frac{€}{\mathrm{~h}}\right]$ & $b_{\mathrm{G}}\left[\frac{1}{\mathrm{MW}} \frac{€}{\mathrm{~h}}\right]$ & $a_{\mathrm{q}}\left[\frac{1}{\mathrm{Mvar}^{2}} \frac{€}{\mathrm{~h}}\right]$ \\
\hline $\mathrm{G} 1$ & 0.010 & 15 & 0.001 \\
\hline $\mathrm{G} 2$ & 0.011 & 30 & 0.001 \\
\hline G3 & 0.012 & 40 & 0.001 \\
\hline RES & 0 & 5 & 0.001 \\
\hline
\end{tabular}

\begin{tabular}{l|l}
\hline Capacity & $200 \mathrm{MWh}$ \\
\hline Maximal power & $100 \mathrm{MW}$ \\
\hline Minimal energy & $20 \mathrm{MWh}$ \\
\hline Maximal energy & $180 \mathrm{MWh}$ \\
\hline Efficiency & $95 \%$ \\
\hline Costs & \\
\hline - discharge & $10 € / \mathrm{MWh}$ \\
\hline - charge & $0 € / \mathrm{MWh}$ \\
\hline
\end{tabular}




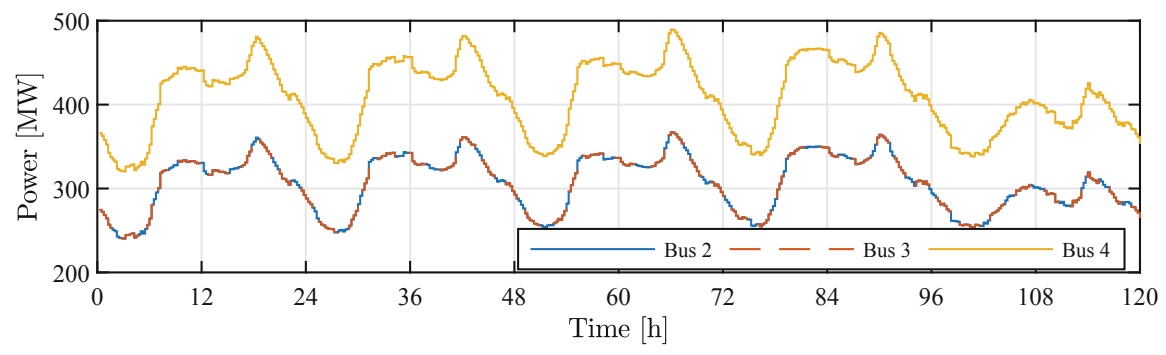

Fig. 6 Load profiles

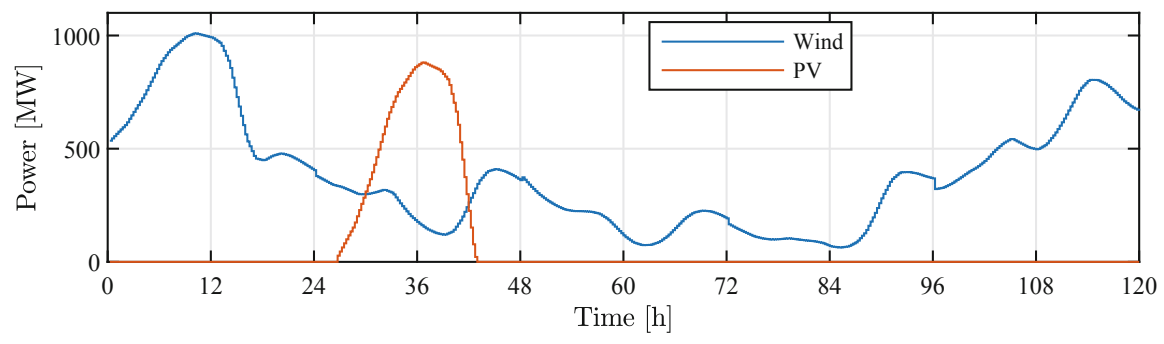

Fig. 7 RES profiles

Acknowledgements The authors kindly acknowledge the support for this work from the German Research Foundation (DFG) under the Project Number LE1432/14-2.

\section{References}

1. C.E. Murillo-Sanchez, R.D. Zimmerman, C.L. Anderson, R.J. Thomas, Decis. Support. Syst. 56, 1 (2013). https://doi.org/10.1016/j.dss.2013.04.006

2. J. Eickmann, C. Bredtmann, A. Moser, in Trends in Mathematics (Springer International Publishing, 2017), pp. 47-63. https://doi.org/10.1007/978-3-319-51795-7_4

3. H. Sharifzadeh, N. Amjady, H. Zareipour, Electr. Power Syst. Res. 146, 33 (2017). https://doi. org/10.1016/j.epsr.2017.01.011

4. A. Fuchs, J. Garrison, T. Demiray, in 2017 IEEE Manchester PowerTech, 2017, pp. 1-6. https:// doi.org/10.1109/PTC.2017.7981181

5. C.E. Murillo-Sanchez, R.D. Zimmerman, C.L. Anderson, R.J. Thomas, IEEE Trans. Smart Grid 4(4), 2220 (2013). https://doi.org/10.1109/tsg.2013.2281001

6. A.J. Lamadrid, D.L. Shawhan, C.E. Murillo-Sánchez, R.D. Zimmerman, Y. Zhu, D.J. Tylavsky, A.G. Kindle, Z. Dar, IEEE Trans. Power Syst. 30(2), 1064 (2015). https://doi.org/10.1109/ TPWRS.2014.2388214

7. Y. Wen, C. Guo, H. Pandzic, D.S. Kirschen, IEEE Trans. Power Syst. 31(1), 652 (2016). https:// doi.org/10.1109/tpwrs.2015.2407054

8. N. Meyer-Huebner, M. Suriyah, T. Leibfried, in 2018 IEEE PES Innovative Smart Grid Technologies Conference Europe (ISGT-Europe) (IEEE, 2018). https://doi.org/10.1109/isgteurope. 2018.8571779 
9. B.H. Kim, R. Baldick, IEEE Trans. Power Syst. 15(2), 599 (2000). https://doi.org/10.1109/59. 867147

10. D.K. Molzahn, F. Dörfler, H. Sandberg, S.H. Low, S. Chakrabarti, R. Baldick, J. Lavaei, IEEE Trans. Smart Grid 8(6), 2941 (2017). https://doi.org/10.1109/TSG.2017.2720471

11. S. Boyd, N. Parikh, E. Chu, B. Peleato, J. Eckstein, Found. Trends Mach. Learn. 3(1), 1 (2011). https://doi.org/10.1561/2200000016

12. T. Erseghe, IEEE Trans. Power Syst. 29(5), 2370 (2014). https://doi.org/10.1109/TPWRS. 2014.2306495

13. T. Erseghe, EURASIP J. Adv. Signal Process. 2015(1), 45 (2015). https://doi.org/10.1186/ s13634-015-0226- $\mathrm{x}$

14. J. Guo, G. Hug, O.K. Tonguz, IEEE Trans. Power Syst. 32(5), 3842 (2017). https://doi.org/10. 1109/TPWRS.2016.2636811

15. N. Meyer-Huebner, M. Suriyah, T. Leibfried, IEEE Trans. Power Syst. 34(4), 2937 (2019). https://doi.org/10.1109/TPWRS.2019.2892240

16. K. Baker, G. Hug, X. Li, in 2012 IEEE Power and Energy Society General Meeting (IEEE, 2012). https://doi.org/10.1109/pesgm.2012.6344712

17. S. Mhanna, G. Verbic, A.C. Chapman, CoRR abs/1704.03647 (2017). http://arxiv.org/abs/ 1704.03647

18. A. Engelmann, T. Mühlpfordt, Y. Jiang, B. Houska, T. Faulwasser, in IFAC-PapersOnLine (20th IFAC World Congress, 2017), vol. 50, pp. 5536-5541. https://doi.org/10.1016/j.ifacol. 2017.08.1095

Open Access This chapter is licensed under the terms of the Creative Commons Attribution 4.0 International License (http://creativecommons.org/licenses/by/4.0/), which permits use, sharing, adaptation, distribution and reproduction in any medium or format, as long as you give appropriate credit to the original author(s) and the source, provide a link to the Creative Commons licence and indicate if changes were made.

The images or other third party material in this chapter are included in the chapter's Creative Commons licence, unless indicated otherwise in a credit line to the material. If material is not included in the chapter's Creative Commons licence and your intended use is not permitted by statutory regulation or exceeds the permitted use, you will need to obtain permission directly from the copyright holder.

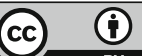

